\title{
Expression invariant face recognition using multi-stage 3D face fitting with 3D morphable face model
}

\begin{abstract}
This paper aims to propose a new fully automated three-dimensional model based, real-time capable approach to recognize facial expressions from visual images of human faces in real time scenario. A multistage 3D fitting algorithm is applied with a morphable model to ensure the high accuracy and speed of the process in addition to eliminating the pose and illumination effects during the recognition process. The idea of the model is to update parameters at each stage in the fitting process. Feature extraction will be done using active appearance model while the feature classification will be done using the tree model to insure a good processing speed. This proposed model will show good results when shape, texture and extrinsic variations occur in the $3 \mathrm{D}$ domain since the combination of multistage fitting algorithm and tree model can enhance the speed and accuracy of the system recognition capabilities. This 3D morphable model algorithm can be widely used for 3D face analysis and $3 \mathrm{D}$ face recognition in real time scenarios.
\end{abstract}

Keyword: 3D morphable model; Expression invariant; Face recognition; Multistage fitting 